\title{
Stakeholder perceptions of misoprostol: a qualitative investigation
}

This article was published in the following Dove Press journal:

International Journal of Women's Health

8 April 2014

Number of times this article has been viewed

\author{
Alessandra N Bazzano' \\ Lea Jones ${ }^{\prime}$ \\ Thoai D Ngo² \\ 'Department of Global Community \\ Health and Behavioral Sciences, Tulane \\ University School of Public Health \\ and Tropical Medicine, New Orleans, \\ LA, USA; ${ }^{2}$ Research, Monitoring \\ and Evaluation Team, Health \\ System Department, Marie Stopes \\ International, London, UK
}

\begin{abstract}
The study aimed to explore perceptions of stakeholders regarding misoprostol use in Cambodia, a setting with high maternal mortality. Semi-structured expert interviews were conducted with 21 participants in the capital, Phnom Penh. The sample included participants involved in providing reproductive health services through international and local health agencies and the pharmaceutical industry. A theme of controversy over the role of misoprostol in the context of reproductive health services emerged, along with a need to reconcile legitimate viewpoints in order to understand the place of misoprostol in the Cambodian reproductive health setting. Understanding stakeholder perspectives on misoprostol can shed light on the drug's role in reproductive health programming where maternal mortality is high and health facilities are still improving.
\end{abstract}

Keywords: maternal mortality, misoprostol, post-partum hemorrhage, medical abortion, unsafe abortion, Cambodia

\section{Introduction}

Access to high quality reproductive health services is of primary concern in Cambodia due to high levels of maternal mortality and morbidity over the last two decades. ${ }^{1-3}$ High utilization of the private sector to meet reproductive health needs, especially for abortion, has complicated efforts to document access and to accurately describe maternal survival there. ${ }^{4-6}$ The Royal Government of Cambodia has assigned a high priority to improving maternal survival, including the development and implementation of the Fast Track Initiative Road Map for Reducing Maternal and Newborn Mortality 2010-2015, ${ }^{7}$ and the devotion of considerable resources to meeting goals identified in the national strategy. The Prime Minister of Cambodia announced in 2010 that the highest health priority in the nation was the reduction of maternal mortality. The Fast Track Initiative's key interventions are ensuring that women deliver in health facilities, have access to high quality emergency services, and utilize family planning methods. These interventions will hopefully contribute to Cambodia's reported decline of morbidity due to unsafe abortions as well as decrease overall incidence of unsafe abortion, which remains high in many low resource settings. ${ }^{8,9}$

Misoprostol is an inexpensive, heat-stable, and widely available prostaglandin that is registered worldwide for both gastric ulcer and obstetric gynecologic use, and may be an important tool in the arsenal of interventions to promote maternal survival. ${ }^{10,11}$ Lifesaving interventions utilizing misoprostol at the community level have been implemented in settings with high levels of maternal morbidity and mortality - particularly where post-partum hemorrhage is prevalent and public facilities under-resourced - and
Correspondence: Alessandra N Bazzano Department of Global Community Health and Behavioral Sciences, Tulane University School of Public Health and Tropical Medicine, 1440 Canal St, New Orleans, LA 70I I2, USA

Tel + I 5049882338

$\mathrm{Fax}+\mathrm{I} 5049883540$

Email abazzano@tulane.edu 
in many countries the drug has been socially marketed through the private sector as part of a medication abortion regimen. ${ }^{12-14}$ While the drug has proved to be a potentially vital resource for reduction of maternal mortality, controversy over the use of the drug has often related to its potential use as an abortifacient in settings with legal, social, or other restrictions on abortion. ${ }^{15}$ Abortion is legal in Cambodia ${ }^{16}$ and misoprostol is registered in the country for treatment of gastric ulcers. In addition, the combination drug Medabon ${ }^{\circledR}$ (mifepristone/misoprostol) is licensed for medical abortion by trained providers and socially marketed through the Concept Foundation (Bangkok, Thailand) and Population Services International (Washington, DC, USA) ${ }^{17}$ Despite its legal status, misoprostol is not part of the public health sector reproductive service strategy. Contraceptive prevalence is relatively low in Cambodia, with $35 \%$ of women using a modern method and $50 \%$ using any method, while a significant number of women are still delivering outside of health facilities - more than half of women in rural areas. ${ }^{3}$ Thus, the use of misoprostol, both for prevention of post-partum hemorrhage and abortion services, could be beneficial for maternal survival.

Although estimates of abortion in the country are difficult to obtain, it is projected that the majority of women seeking abortions obtain them through the private sector each year, including medication abortion using misoprostol. ${ }^{18}$ Nearly $80 \%$ of women who had an abortion did so in a private sector clinic or in a private home setting in $2010 .{ }^{3}$ Private providers are widely used by the Cambodian population and a pattern of sequential care seeking is common, with drug retailers often being the first point of contact for health and reproductive care. Meessen et al used household surveys to investigate choice of medical provider in Cambodia's pluralistic health system and found that drug retailers were utilized most frequently followed by private practitioners and finally public providers. ${ }^{19}$ Ozawa and Walker also investigated provider preference, considering the role of trust in deciding between public or private providers. ${ }^{20}$ The results suggested that the interpersonal relationship between patient and provider influences care seeking in Cambodia - in effect, decisions were based on individual relationship rather than the perception of the organization or facility in which the provider worked. ${ }^{20}$

In one study of unmarried migrant Cambodian women, self-induced abortion was the birth control of choice. ${ }^{4}$ Participants in that study reported stigma and judgment encountered at health clinics and preferred to seek abortion drugs from pharmacists and street vendors to protect their confidentiality. Some providers in the same study described abortion as an opportunity to supplement their income, and it is possible that not all women who were given medication abortion received proper instructions or gestational screening needed for safe use of the method.

In a study assessing regional availability of misoprostol, Asia was found to have a large and growing market for misoprostol-only drugs and high registration for obstetricgynecologic use. ${ }^{21}$ Of the world regions, Asia has not only the largest selection of misoprostol brands, but provides them at the lowest cost. ${ }^{21}$ However, in countries where misoprostol is widely available without proper instruction and supervision of health providers, there are concerns about the potential for an increase in maternal-fetal complications and misuse, potentially impacting maternal health. ${ }^{22-24}$

In order to understand how the potential controversies over the use of misoprostol may be impacting its use within existing reproductive health services, particularly with regard to the private sector, this study set out to gather perceptions of Cambodian and international stakeholders involved in reproductive health service programming.

\section{Methods}

This study assessed perceptions about misoprostol through three groups of stakeholders in reproductive health-1) program managers and staff of international organizations, 2) program managers and staff of local non-governmental health organizations, and 3) pharmaceutical industry personnel - around the role of misoprostol in private sector reproductive health in Cambodia. Methods included interviews with participants and analysis of country level reports on reproductive health produced by the Ministry of Health and non-governmental organizations. Semi-structured expert interviews were conducted with 21 participants from July to August 2011. Data was coded and themes were analyzed using $\mathrm{NVivo}^{\circledR}$ qualitative analysis software (version 10:2012; QSR International Pty Ltd, Doncaster, VIC, Australia). Interviews were transcribed by the interviewer. The interviews were conducted by an experienced researcher in English (due to fluency by all participants) who analyzed the data, ensured consistency of data with key informants, and kept all transcripts secure. Informed consent was obtained from all participants interviewed during the study and ethics approval to interview individuals was granted by the Internal Review Board of the lead author's institution at the time the study was conducted.

\section{Results}

\section{Private sector availability}

Misoprostol is widely available in Cambodia and is sold in private pharmacies, though primarily it is found in 
combination with mifepristone as a medical abortion product socially marketed through the Concept Foundation and Population Services International. ${ }^{17}$ There are several thousand pharmacies in Phnom Penh alone and misoprostol-only drugs were reported to be widely available, costing around $\$ 7$ for the premium brand, Cytotec ${ }^{\circledR}$ (Pfizer Inc, New York, NY, USA). Chinese brands were described as also being very popular. Participants stated that pharmacies will often decide if they are comfortable selling the product to a particular individual on the basis of whether there is concern the pharmacy might be censured for sale of the product without a prescription.

\section{Controversy and concerns}

The dominant theme that emerged from interviews with stakeholders was controversy over the use of misoprostol in Cambodia. The overwhelming majority of participants interviewed noted that although misoprostol is available in the country, it has not been prioritized in the public sector as a tool to reduce maternal mortality. Analysis of the Cambodia Safe Motherhood Clinical Management Protocols ${ }^{25}$ and the Emergency Obstetric Care Strategy 2010-2015 ${ }^{26}$ confirmed that misoprostol is not included or mentioned at all as an uterotonic agent for prevention and treatment of post-partum hemorrhage or for medical abortion. However, the government has approved a combination misoprostol plus mifepristone drug, Medabon ${ }^{\circledR}$ (Concept Foundation, Bangkok, Thailand), only for use by licensed providers for medication abortion in clinics. ${ }^{17}$

Participants unanimously agreed that historically misoprostol has caused controversy in Cambodia and, as a result of that, is not a high priority in the overall strategy for reproductive health at present. One of the reasons for controversy cited by the majority of participants was a case in 2008 of maternal death as the result of a missed ectopic pregnancy for which the provider had prescribed misoprostol for medical abortion. Although the case was clearly identified as a missed ectopic pregnancy and not a death due to the use of misoprostol, it caused concern among policymakers. One informant stated that "even though it was the ectopic pregnancy which was responsible for the case, and international investigation was completed, the damage was done for misoprostol" (reproductive health program manager). All study participants reported a degree of concern in discussing the topic of misoprostol as it had become a sensitive issue in the context of public and private health services as a result of the case. One participant stated, "[misoprostol] is important but is probably not on anyone's agenda right now".

A second controversy regarding misoprostol that emerged from interview data was the debate over whether prioritizing misoprostol through policies or programs could undermine the national effort to strengthen safe delivery services. Some participants noted that the country-wide focus on strengthening facility-based delivery, including provision of oxytocin as an uterotonic and the routine provision of active management of the third stage of labor, lessened the need for misoprostol as a tool to prevent post-partum hemorrhage. One informant stated, "due to a spectacular rise in facility based births over the last several years, misoprostol may be a moot point for prevention of post-partum hemorrhage" (staff of an international aid organization). Another noted, "oxytocin is available at community level facilities and approximately $90 \%$ of women are receiving active management of the third stage of labor" (program officer for a multilateral donor).

Furthermore, one participant related the reluctance on the part of the national policy makers to promote the use of misoprostol to fear that it could jeopardize facility delivery if women and families preferentially used misoprostol in the home or community, rather than accessing public services. The participant went on to note that "roads and transportation are becoming more accessible and many programs are in place to reduce the burden of post-partum hemorrhage without needing to make misoprostol available more widely". One staff member of a local non-governmental organization stated that in the past, international organizations had pressed national policymakers to introduce misoprostol at the community level, but that the effort failed due to divergent opinions on the impact it could have on facility-based treatment using skilled attendants.

The general consensus among those interviewed, however, was that misoprostol was an important option in the arsenal against maternal death and should be given more attention at the national level. According to one participant, "women are still dying in the villages during childbirth, [the country] could still reduce the maternal mortality rate if misoprostol was being used at the community level" (staff member of an international non-governmental). One interviewee pointed out that although the Cambodia Demographic and Health Survey data show reductions in maternal mortality from 2005 to 2010 , the ratio is likely to be underreported due to problems with accurate reporting of deaths at community and facility level and due to high utilization of private providers, especially for abortion.

\section{Concerns over private sector use}

Another perception of several participants was that private providers might prefer misoprostol not be available because of the loss of income from surgical abortions. Medication abortion, 
induced either by misoprostol alone or in combination with mifepristone, and whether supervised or not, is much less expensive for patients than vacuum aspiration abortion - costing on average between \$5-\$7 for medication abortion in Phnom Penh versus \$30-\$200 for surgical abortion. One study participant described the problem of a public health system with low salaries for health providers that may encourage them to steer women towards more costly surgical abortion in private clinics.

Participants from pharmaceutical entities noted that the Cambodian drug market is a saturated and competitive one within a growing economy and has the added complication of very porous national borders that allow smuggling of pharmaceutical products from nearby countries. Interviewees noted that there are copycat drugs and a multitude of outlets that sell products such as misoprostol without proper counseling. It was also noted that pharmacies are starting to be regulated more strictly and in the future unregistered pharmacies and drug sellers may be closed down due to government enforcement.

According to participants, there are other issues that may hamper the use of misoprostol and impact on demand for the product. Pharmaceutical informants noted that doctors often dispense directly from their offices and pharmacy owners also preferentially recommend products that are under promotion by pharmaceutical distributors in order to ensure the best profit margins. Other participants noted that some doctors prescribe medicines using codes so that patients cannot learn the names of drugs and request them at pharmacies and others use drug cocktails in order to prevent patients from self-medication. Demand for products is based on awareness of the product by consumers. Awareness can be generated by pharmacy owners, providers, or word of mouth.

One interesting point mentioned by pharmaceutical informants was that Cambodian clients may consider Chinese or Indian pharmaceutical products to be inferior to Korean, Philippine, and especially European ones, making cost an issue. Additionally, wholesalers of pharmaceutical products may be a key point of information for consumers and are a key entry point into the markets.

\section{Discussion}

In settings where facility-based care is improving rapidly but maternal mortality from post-partum hemorrhage and unsafe abortion remain relatively high, misoprostol could have a role in promoting maternal survival. Controversies and legitimate concerns on the part of stakeholders provide some answers for the omission. While misoprostol has been successfully used to address maternal mortality from post-partum hemorrhage, Cambodian stakeholders mentioned concerns related to possible decreases in health facility delivery. Misoprostol has been used in Nepal without compromising increases in facility-based delivery where oxytocin is available. ${ }^{13}$ And, although the maternal mortality ratio has decreased significantly in Cambodia, further declines could be made as nearly $30 \%$ of women are still giving birth without a skilled attendant and $46 \%$ outside of a health facility particularly in rural areas with low levels of educational attainment. ${ }^{3}$

Regarding use of misoprostol for medication abortion, stakeholders stated that some providers persuade women to opt for surgical abortion due to higher profits reducing the demand for misoprostol as it is used with mifepristone for medical abortion. However, a recent study suggested that the cost of the two procedures may be equivalent in settings where women require follow-up treatment after medication ${ }^{27}$ and another study noted that women undergoing medication abortion reported more negative experiences of care and lower acceptability, ${ }^{28}$ suggesting that factors unrelated to provider preference may be at work.

The study had some important limitations. A much larger, quantitative study would have been necessary to understand the exact distribution, prescribing practices, and purchasing behaviors of misoprostol, and thus was beyond the scope of this study. Misoprostol is available in the country and is sold in pharmacies primarily in combination with mifepristone, which complicates efforts to understand the role of misoprostol-only drugs.

\section{Conclusion}

Ultimately, the extent to which misoprostol is utilized in a particular reproductive health setting will depend on many factors, including demand for the drug from providers and individuals, and the historical context of use of the drug. Documenting the legitimate concerns among stakeholders involved in reproductive health programming and services can shed light on reasons for not including misoprostol in a national strategy when maternal survival can be improved.

\section{Acknowledgments}

The authors acknowledge Marie Stopes International for partial funding of this research.

\section{Disclosure}

All authors had access to the data, commented on subsequent drafts, and approved the final submitted version. All authors read and approved the final manuscript. The sponsor of the study (Marie Stopes International) had no role in study design, data collection, data analysis, data interpretation, or 
writing of this paper. The manuscript contents are the sole responsibility of the authors and do not necessarily reflect the views of the sponsor.

\section{References}

1. Cambodia National Institute of Public Health, Cambodia National Institute of Statistics, ORC Macro. Cambodia Demographic and Health Survey 2005. Calverton, MD: Measure DHS; 2006. Available from: http://www.measuredhs.com/pubs/pdf/FR185/FR185[April-27-2011]. pdf. Accessed January 16, 2014.

2. Cambodia National Institute of Statistics, Cambodia Ministry of Planning and Directorate General for Health, Cambodia Ministry of Health, ORC Macro. Cambodia Demographic and Health Survey 2000. Calverton, MD: Measure DHS; 2001. Available from: http://www. measuredhs.com/pubs/pdf/FR124/FR124.pdf. Accessed January 16, 2014.

3. Cambodia National Institute of Statistics, Directorate General for Health, Cambodia Ministry of Health, ICF Macro. Cambodia Demographic and Health Survey 2010. Calverton, MD: Measure DHS; 2011. Available from: http://www.measuredhs.com/pubs/pdf/FR249/FR249.pdf. Accessed January 16, 2014.

4. Hegde SS, Hoban E, Nevill A. Unsafe abortion as a birth control method: maternal mortality risks among unmarried Cambodian migrant women on the Thai-Cambodia border. Asia Pac J Public Health. 2012;24(6): 989-1001.

5. Hemings J, Rolfe B. Abortion in Cambodia. Care Seeking for Abortion and Family Planning Services: Findings from a PEER Study, Conducted with Women in Phnom Penh and Kandal Provinces. London: Options Consultancy Services; 2008. Available from: http://www.options.co.uk/ images/stories/resources/peer/cambodia_2007_abortion.pdf. Accessed January 16, 2014.

6. Fetters T, Vonthanak S, Picardo C, Rathavy T. Abortion-related complications in Cambodia. BJOG. 2008;115(8):957-968.

7. Cambodia Ministry of Health. Fast Track Initiative Road Map for Reducing Maternal and Newborn Mortality 2010-2015. Phnom Penh Ministry of Health; 2010. Available from: http://www.unfpa.org/ sowmy/resources/docs/library/R123_MOHCambodia_2010_Fast_ TrackInitiativeRoadMapforReducingMaternalandNewbornMortality. pdf. Accessed January 16, 2014.

8. Fetters T, Barnett S, Chourn T, et al. Making a Difference: A National Assessment of Abortion Care in Cambodian Public Health Facilities, 2005 and 2010. Chapel Hill, NC: Ipas; 2010.

9. Sedgh G, Singh S, Shah IH, Ahman E, Henshaw SK, Bankole A. Induced abortion: incidence and trends worldwide from 1995 to 2008. Lancet. 2012;379(9816):625-632.

10. Prata N, Hamza S, Gypson R, Nada K, Vahidnia F, Potts M. Misoprostol and active management of the third stage of labor. Int J Gynaecol Obstet. 2006;94(2):149-155.

11. Prata N, Gessessew A, Abraha AK, Holston M, Potts M. Prevention of postpartum hemorrhage: options for home births in rural Ethiopia. Afr J Reprod Health. 2009;13(2):87-95.

12. Rajbhandari S, Hodgins S, Sanghvi H, McPherson R, Pradhan YV, Baqui AH. Expanding uterotonic protection following childbirth through community-based distribution of misoprostol: operations research study in Nepal. Int J Gynaecol Obstet. 2010;108(3):282-288.
13. Chandhiok N, Dhillon BS, Datey S, Mathur A, Saxena NC. Oral misoprostol for prevention of postpartum hemorrhage by paramedical workers in India. Int J Gynaecol Obstet. 2006;92(2):170-175.

14. Derman RJ, Kodkany BS, Goudar SS, et al. Oral misoprostol in preventing postpartum haemorrhage in resource-poor communitites: a randomised controlled trial. Lancet. 2006;368(9543):1248-1253.

15. Nations MK, Misago C, Fonseca W, Correia LL, Campbell OM. Women's hidden transcripts about abortion in Brazil. Soc Sci Med. 1997;44(12):1833-1845.

16. Cambodian laws: Kram abortion [webpage on the Internet]. Phnom Penh: Cambodian National Assembly; 1997. Available from: http:// www.asianlii.org/kh/legis/laws/ka125/. Accessed January 16, 2014.

17. Medabon ${ }^{\circledR}$ : key talking points [webpage on the Internet]. Available from: http://www.medabon.info/key.php. Bangok: Concept Foundation; 2009. Accessed December 19, 2012.

18. Fetters T, Rathavy T, Sherman J, et al. Ready or Not? A National Needs Assessment of Abortion Services in Cambodia. Chapel Hill, NC: Ipas; 2007. Available from: http://www.ipas.org/ /media/Files/Ipas\%20 Publications/ABORCAME07.ashx?utm_source=resource\&utm_ medium $=$ meta\&utm_campaign $=$ ABORCAME07. Accessed January 16, 2014.

19. Meessen B, Bigdeli M, Chheng K, et al. Composition of pluralistic health systems: how much can we learn from household surveys? An exploration in Cambodia. Health Policy Plan. 2011;26(Suppl 1): i30-i44.

20. Ozawa S, Walker DG. Comparison of trust in public vs private health care providers in rural Cambodia. Health Policy Plan. 2011;26(Suppl 1): i20-i29.

21. Fernandez MM, Coeytaux F, de Leon RG, Harrison DL. Assessing the global availability of misoprostol. Int J Gynaecol Obstet. 2009;105(2): 180-186.

22. Pastuszak AL, Schuler L, Speck-Martins CE, et al. Use of misoprostol during pregnancy and Mobius' syndrome in infants. $N$ Engl $J$ Med. 1998;338(26):1881-1885.

23. Saha PK, Prabhakar S, Goel P, Tandon R. Misuse of abortion pill by unauthorized persons is a serious concern in India. J Obstet Gynaecol Res. 2010;36(6):1265-1266.

24. Gonzalez CH, Marques-Dias M, Kim CA, et al. Congenital abnormalities in Brazilian children associated with misoprostol misuse in first trimester of pregnancy. Lancet. 1998;351(9116):1624-1627.

25. Cambodia Ministry of Health Reproductive Health Program. Safe Motherhood Clinical Management Protocols Health Centers. Phnom Penh: Ministry of Health; 2010. Available from: http://www.moh.gov. $\mathrm{kh} /$ files/mch/Safe\%20Motherhood\%20for\%20HC\%20English.pdf. Accessed January 16, 2014.

26. Cambodia Ministry of Health. Cambodia EmONC Improvement Plan for Implementation January 2010 - December 2015. Phnom Penh: Ministry of Health; 2009. Available from: http://www.unfpa.org/sowmy/ resources/docs/library/R124_MOHCambodia_2009_Final_EmONC_ Improvement_Plan_March2010.pdf. Accessed January 16, 2014.

27. Xia W, She S, Lam TH. Medical versus surgical abortion methods for pregnancy in China: a cost-minimization analysis. Gynecol Obstet Invest. 2011;72(4):257-263.

28. Howie FL, Henshaw RC, Naji SA, Russell IT, Templeton AA. Medical abortion or vacuum aspiration? Two-year follow up of a patient preference. Br J Obstet Gynaecol. 1997;104(7):829-833.
International Journal of Women's Health

\section{Publish your work in this journal}

The International Journal of Women's Health is an international, peerreviewed open-access journal publishing original research, reports, editorials, reviews and commentaries on all aspects of women's healthcare including gynecology, obstetrics, and breast cancer. The manuscript management system is completely online and includes

\section{Dovepress}

a very quick and fair peer-review system, which is all easy to use. Visit http://www.dovepress.com/testimonials.php to read real quotes from published authors. 